\title{
Indentation of aluminium foam at low velocity
}

\author{
Xiaopeng $\mathrm{Shi}^{1}$, Yinggang Miao ${ }^{1}$, Shuangyan $\mathrm{Liu}^{1}$, Yulong $\mathrm{Li}^{1,}$, a and Guoxing $\mathrm{Lu}^{2}$ \\ ${ }^{1}$ School of Aeronautics, Northwestern Polytechnical University, Xi' an 710072, China \\ ${ }^{2}$ Faculty of Science, Engineering and Technology, Swinburne University of Technology, Hawthorn, Vic 3122, Australia
}

\begin{abstract}
The indentation behaviour of aluminium foams at low velocity $(10 \mathrm{~m} / \mathrm{s} \sim 30 \mathrm{~m} / \mathrm{s})$ was investigated both in experiments and numerical simulation in this paper. A flat-ended indenter was used and the force-displacement history was recorded. The Split Hopkinson Pressure bar was used to obtain the indentation velocity and forces in the dynamic experiments. Because of the low strength of the aluminium foam, PMMA bar was used, and the experimental data were corrected using Bacon's method. The energy absorption characteristics varying with impact velocity were then obtained. It was found that the energy absorption ability of aluminium foam gradually increases in the quasi-static regime and shows a significant increase at $\sim 10 \mathrm{~m} / \mathrm{s}$ velocity. Numerical simulation was also conducted to investigate this process. A 3D Voronoi model was used and models with different relative densities were investigated as well as those with different failure strain. The indentation energy increases with both the relative density and failure strain. The analysis of the FE model implies that the significant change in energy absorption ability of aluminium foam in indentation at $\sim 10 \mathrm{~m} / \mathrm{s}$ velocity may be caused by plastic wave effect.
\end{abstract}

\section{Introduction}

As a lightweight energy-absorbing material, aluminium foam has been widely used in automotive industry and aerospace engineering [1] Researchers showed more and more interests in mechanical properties of this material, especially the energy-absorbing mechanism. When this material is frequently used in blast-protected devices [2], indentation becomes a potential mode of failure. Ramachandra et al. [3,4] experimentally investigated the indentation behaviour of an aluminium foam under dynamic loads. In their research, drop-tower experiments were used to investigate the energy-absorption ability of aluminium foams under a velocity of $3 \sim 30 \mathrm{~m} / \mathrm{s}$. It was found that there is significant change in energy absorption ability of aluminium foam in indentation at $\sim 10 \mathrm{~m} / \mathrm{s}$. But the experimental data at velocity of $3 \sim 30 \mathrm{~m} / \mathrm{s}$ were limited, and the indentation velocity was not constant in droptower tests. Besides, the role of the tearing energy in indentation at low velocity is still unknown. In this paper, the SHPB (Split Hopkinson Pressure bar) was used in indentation tests and an FEM model was established to investigate the energy absorption ability at the velocity of $10 \sim 30 \mathrm{~m} / \mathrm{s}$. Empirical formulas were obtained to describe the relationship between the indentation energy and the indentation velocities.

\section{Materials and method}

\subsection{Materials and specimens}

Commercial closed-cell aluminium foam, ALPORAS, was used in this study. This foam was made from aluminium with $1.5 \%$ of $\mathrm{Ca}$ and $1.5 \%$ of $\mathrm{Ti}$ as well. The average pore size is $\sim 3.5 \mathrm{~mm}$ and the nominal relative density of this material is $\sim 0.09$ as provided by the manufacturer. A typical macroscopic view of this material is shown in Fig. 1.

The dynamic indentation tests were conducted on blocks of $20 \times 35 \times 50 \mathrm{~mm}$. A steel plate with thickness of $2 \mathrm{~mm}$ was used as indenter and was attacthed to the end of incident bar of SHPB system. To eliminate friction between the specimen and the indenter, the shank of indenter was chamfered at a small angel. The experimental set-up is shown in Fig. 2.

The projected area of the indenter is $20 \mathrm{~mm} \times$ $20 \mathrm{~mm}$, which covers $\sim 30$ cells to ensure the measured data represent the average response of this material. For comparison, the dynamic compression specimen is designed as a $20 \times 20 \times 20 \mathrm{~mm}$ block. Both indentation and compression specimen are shown in Fig. 3.

\subsection{Experimental method}

SHPB system was used in both the indentation and compression experiments. The conventional SHPB analysis is based on the one-dimensional elastic wave propagation assumption. The incident pulse $\varepsilon_{i}(t)$, the reflected pulse $\varepsilon_{r}(t)$ and the transmission pulse $\varepsilon_{t}(t)$ can be recorded by the strain gauges attached to the incident bar and the transmission bar. According to one-dimensional stress wave theory, these signals can be shifted to bar-specimen interfaces. Thus, the velocities and the forces at both faces of the sample can be obtained from the following equations:

$$
\begin{gathered}
V_{\text {input }}=C_{0}\left[\varepsilon_{i}(t)-\varepsilon_{r}(t)\right] \\
V_{\text {output }}=C_{0} \varepsilon_{t}(t)
\end{gathered}
$$

\footnotetext{
${ }^{a}$ Corresponding author: liyulong@nwpu.edu.cn
}

This is an Open Access article distributed under the terms of the Creative Commons Attribution License 4.0, which permits unrestricted use, distribution, and reproduction in any medium, provided the original work is properly cited. 


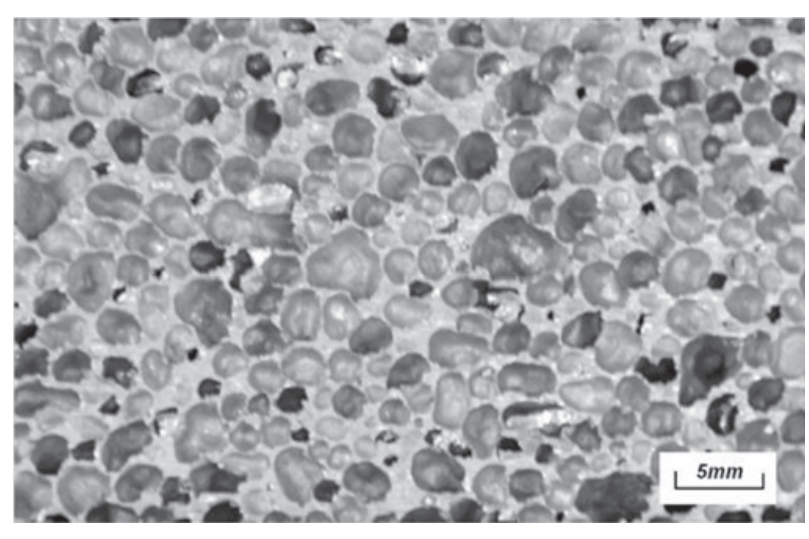

Figure 1. Macroscopic view of ALPORAS foam.

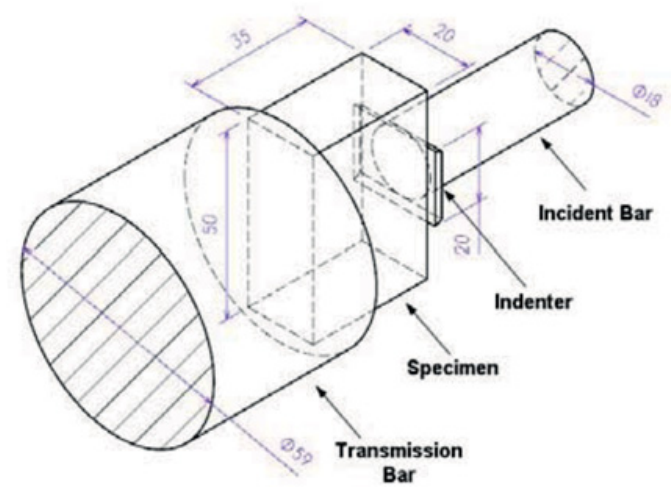

Figure 2. Sketch of the set-up for dynamic indentation test. The dimensions are in $\mathrm{mm}$.
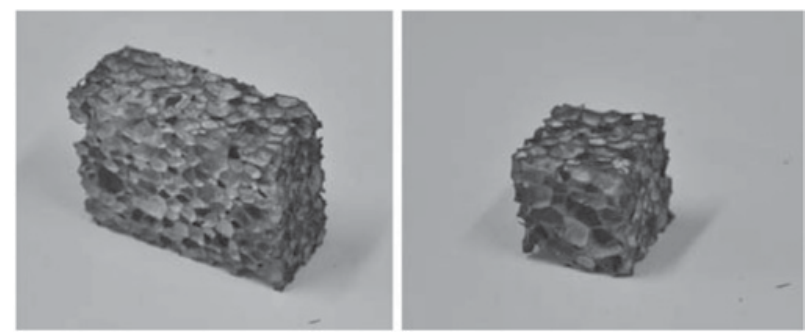

Figure 3. The specimen for indentation test (left) and the specimen for compression test (right).

$$
\begin{gathered}
F_{\text {input }}=E S_{i}\left[\varepsilon_{i}(t)+\varepsilon_{r}(t)\right] \\
F_{\text {output }}=E S_{t} \varepsilon_{t}(t)
\end{gathered}
$$

where $V_{\text {input }}, V_{\text {output }}, F_{\text {input }}, F_{\text {output }}$ are particle velocities and forces at the interfaces, $C_{0}, E, S_{i}$ and $S_{t}$ are, respectively, the longitudinal wave speed in the pressure bars, Young's modulus, the cross-sectional area of incident bar and the cross-sectional area of the transmission bar.

Due to the low strength of cellular materials, the signals from strain gauges on metallic bars would be weak, which leads to a low signal-noise ratio. PMMA bars were used in this study to avoid this problem. The incident bar is $2 \mathrm{~m}$ in length with a diameter of $18 \mathrm{~mm}$ and the transmission bar is $1 \mathrm{~m}$ in length with a diameter of $59 \mathrm{~mm}$. However, as PMMA is a viscoelastic material and the wave dispersion effect cannot be neglected when stress wave propagates in the bar, the signals need correction before being used. Bacon [5] developed an experimental method to correct the dispersion effect. If the lateral motion can be neglected in a straight cylindrical bar, the normal stress $\sigma(x, t)$ and the longitudinal strain $\varepsilon(x, t)$ are related to the axial displacement $u(x, t)$ at cross-section $x$ and at time $t$ by

$$
\begin{aligned}
\frac{\partial \sigma(x, t)}{\partial x} & =\rho \frac{\partial^{2} u(x, t)}{\partial t^{2}} \\
\varepsilon(x, t) & =\frac{\partial u(x, t)}{\partial x} .
\end{aligned}
$$

Equations (5) and (6) can be written in one equation in the Fourier domain:

$$
\frac{\partial^{2} \tilde{\sigma}(x, \omega)}{\partial x^{2}}=-\rho \omega^{2} \tilde{\varepsilon}(x, \omega)
$$

where $\rho, \omega, \tilde{\sigma}(x, \omega)$ and $\tilde{\varepsilon}(x, \omega)$ denote the mass density of the bar, the angular frequency, the Fourier transforms of the stress and strain, respectively.

Then, the linear viscoelastic behavior of the material can be expressed as follows:

$$
\tilde{\sigma}(x, \omega)=E^{*}(\omega) \tilde{\varepsilon}(x, \omega)
$$

where $E^{*}(\omega)$ is the complex Young's modulus. From Eqs. (7) and (8), the one-dimension equation of axial motion of a viscoelatic bar can be written as follows:

$$
\left(\frac{\partial^{2}}{\partial x^{2}}-\gamma^{2}\right) \tilde{\varepsilon}(x, \omega)=0
$$

where the propagation coefficient $\gamma$ can be expressed as follows:

$$
\gamma^{2}=-\frac{\rho \omega^{2}}{E^{*}(\omega)} .
$$

The general solution for equation (9) is:

$$
\tilde{\varepsilon}(x, t)=\tilde{P}(\omega) e^{-\gamma x}+\tilde{N}(\omega) e^{\gamma x}
$$

where the $\tilde{P}(\omega)$ and $\tilde{N}(\omega)$ are the Fourier transforms of the strains at $x=0$ due to the waves traveling in the directions of increasing and decreasing $x$, respectively.

To determine the propagation coefficient $\gamma$, an experiment was conducted. A strain guage was attached in the middle of the incident bar where $x$ was set 0 . The incident bar was impacted by a very short projectile at its left end with its right end $(x=d)$ free. Two strain signals without superpostion was recorded as $\varepsilon_{1}(t)$ and $\varepsilon_{2}(t)$, which refers to the incident wave and the reflected wave, respectively. According to Eq. (11):

$$
\tilde{\varepsilon}_{1}=\tilde{P} \text { and } \tilde{\varepsilon}_{2}=\tilde{N} .
$$

Since the right end of the incident bar is free, the strain at the right end is 0 :

$$
\tilde{P} e^{-\gamma d}+\tilde{N} e^{\gamma d}=0 .
$$

Then the propagation coefficient $\gamma$ can be solved as:

$$
e^{-\gamma 2 d}=-\frac{\tilde{\varepsilon}_{1}(\omega)}{\tilde{\varepsilon}_{2}(\omega)} .
$$




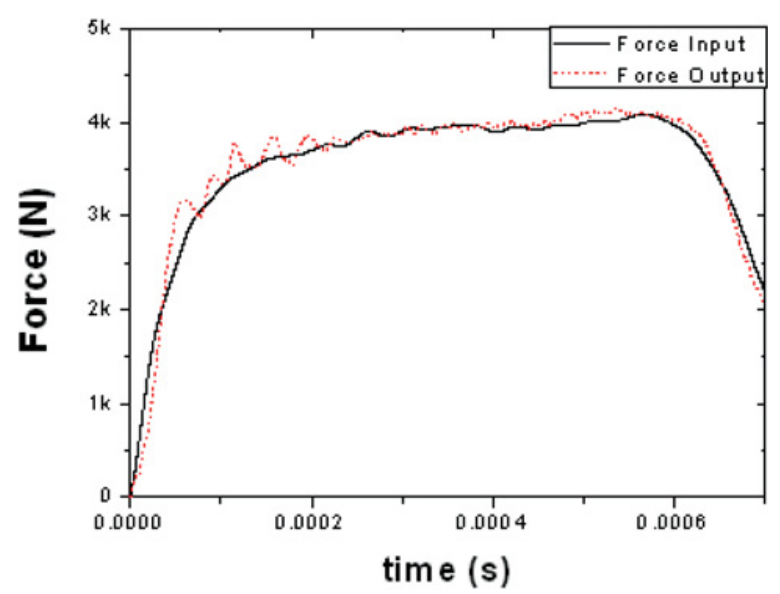

Figure 4. Comparision of input and output forces.

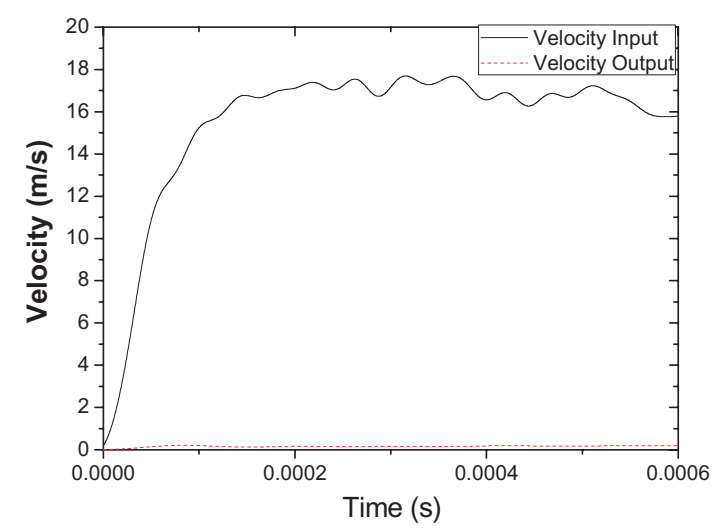

Figure 5. Velocities in a dynamic indentation test using SHPB.

A MATLAB program using the above method was written to correct the experiment result. To verify the correction result, a SHPB test without specimen was performed. Using Eqs. (3) and (4), the input and output forces can be obtained and these forces should be equal. Figure 4 shows the comparition bewteen these two forces and the result indicates that the correction method can be used in our study.

\section{Experimental result and discussion}

\subsection{Compression and indentation forces}

A typical pair of velocity histories of the dynamic indentation test is shown in Fig. 5. The particle velocity at the end of the transmission bar is nearly zero in the test. For velocity of the incident bar, there is a plateau of a duration of $\sim 0.0005 \mathrm{~s}$, which means the impact velocity is constant.

Both indentation and compression tests were conducted and typical curves of normalized force versus normalized indentation depth (compression depth for compression tests) are shown in Fig. 6 and Fig. 7 Note that the force $F$ was normalized with respect to the contact area between the indenter and specimen. The normalized indentation depth was the indentation depth or compression depth $d$ divided by the thickness of the specimen $L$.

Deshpande and Fleck [6] tested Alulight and Duocel foams but no velocity sensitivity was observed. While in

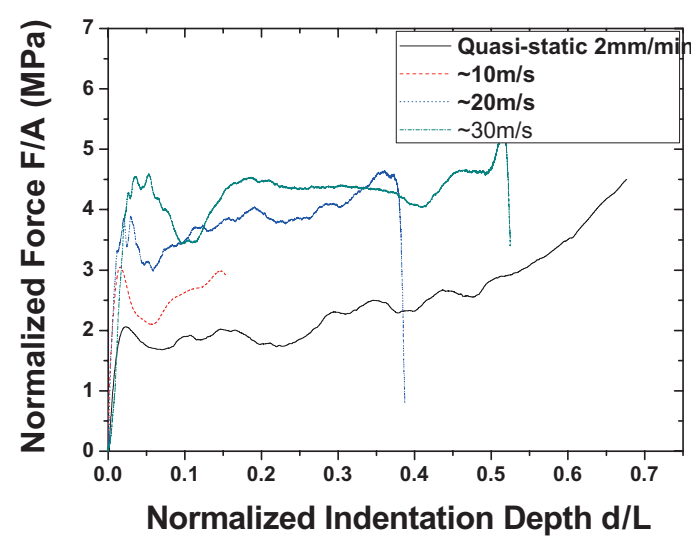

Figure 6. Normalized force vs. Normalized indentation depth for dynamic and quasi-static tests.

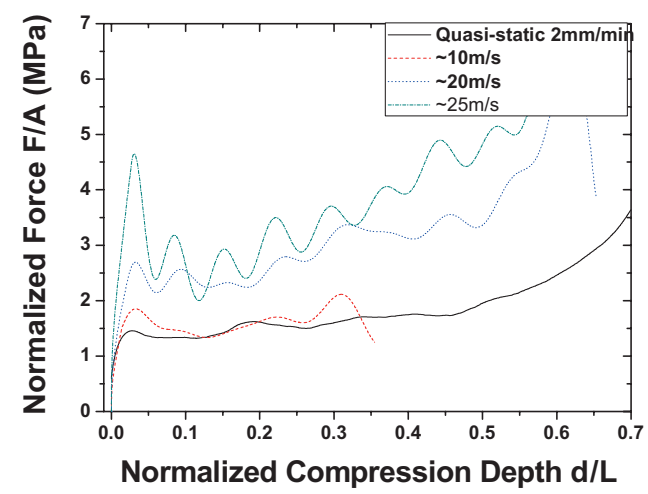

Figure 7. Normalized force vs. Normalized compression depth for dynamic and quasi-static tests.

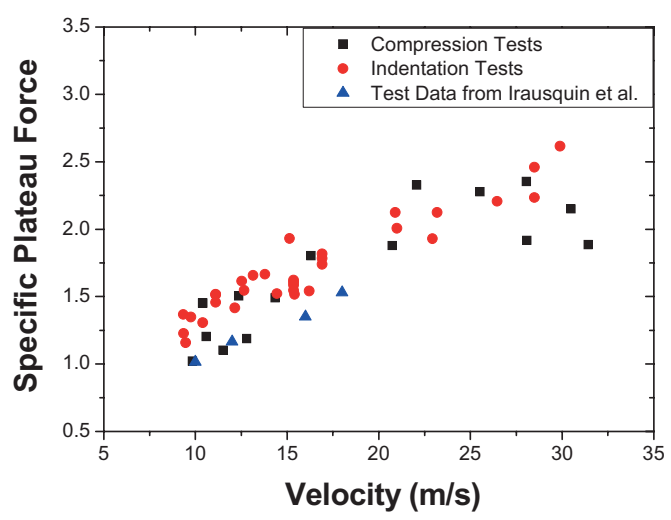

Figure 8. Specific force vs. Loading velocity in the tests.

this research, in both indentation tests and conpression tests, the plateau force shows great velocity sensitivity. Compared with the case of quasi-static loading, the plateau force in indentation at $\sim 30 \mathrm{~m} / \mathrm{s}$ velocity is nearly doubled. Note that the plateau forces in indentation tests are higher than in compression tests due to the forces needed to tear the materials beside the indenter. To show the relationship between the normalized plateau force and the loading velocity, the dynamic plateau forces diveded by quasi-stiac value of all specimens are plotted in Fig. 8, as well as the data from Irausquin et al. [7] They evaluated the dynamic behaviour of closed-cell aluminum foam ALPORAS $10 \%$ using SHPB. 


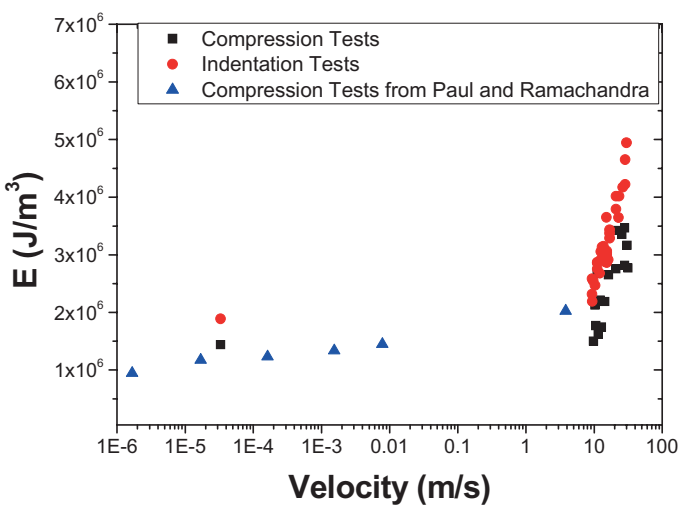

Figure 9. Energy absorbed per unit displaced volume, E vs. the displacement velocity.

In both our tests and Irausquin's tests, there is a significant increase in the specific plateau forces with the increasing velocity between $10 \mathrm{~m} / \mathrm{s} \sim 30 \mathrm{~m} / \mathrm{s}$. As the the cell wall material is mainly made up of aluminum, which has no obvious rate sensitivity, the cell wall material cannot lead to this increase. On the other hand, according to Gibson and Ashby [8], Dashpande and Fleck's [6] research, the contribution of gas trapped in cells is so small as to be negligible for this increase. Microinertia effects of the cell walls [7,9] or the plastic wave [10] may cause this phenomenon. Further discussion will be conducted in FEM analysis.

\subsection{Variation of Energy}

The energy absorbed per unit displaced volume, E, is plotted as a function of the indenter velocity in Fig. 9. Quasi-static experiment result is also added. For comparison, Paul and Ramachandra's [4,11] compression tests results are plotted. Note that the size of indenter which Ramachandra used is different from this research, their indentation tests results are not presented here. For a given velocity within the range of $10^{-6} \mathrm{~m} / \mathrm{s} \sim 30 \mathrm{~m} / \mathrm{s}$, E for indentation is significantly larger than the corresponding value for compression due to the mechanisms of tear and shear involved in indentation. And it can be observed that E varies gradually with the velocity of up to $\sim 10 \mathrm{~m} / \mathrm{s}$ and it increases dramatically above a velocity of $\sim 10 \mathrm{~m} / \mathrm{s}$. This phenomenon implies different mechanism domainates in these two ranges. As researched by Paul [11], the gradual increase of $\mathrm{E}$ or plateau stress is due to the microinertia effects of the cell wall, another mechanism shoul be added for the dramatically increase above a velocity of $\sim 10 \mathrm{~m} / \mathrm{s}$.

To observe the variation of energy absorbed above the velocity of $10 \mathrm{~m} / \mathrm{s}$ clearly, Fig. 10 is presented. The energy absorbed per unit volume by indentation can be written as follows:

$$
E_{\text {ind }}=E_{\text {com }}+E_{\text {tear }}
$$

where $E_{\text {com }}$ and $E_{\text {tear }}$ are energy absorbed per unit volume by compression tests and by indenter tearing the material beside itself. Equation (15) is correct only if the plastic deformation area near the tearing surface is so small that the plastic energy in this area can be neglected. Figure 11 shows the typical specimen after indentation.

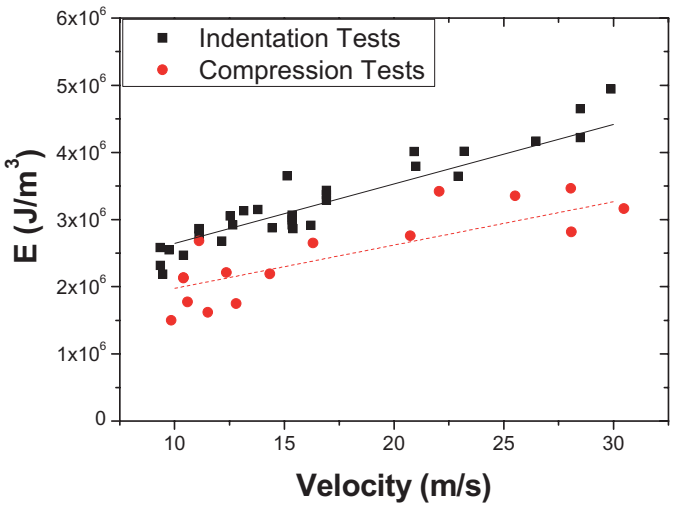

Figure 10. Energy absorbed per unit displaced volume, E vs. the displacement velocity at $10 \mathrm{~m} / \mathrm{s}-30 \mathrm{~m} / \mathrm{s}$.

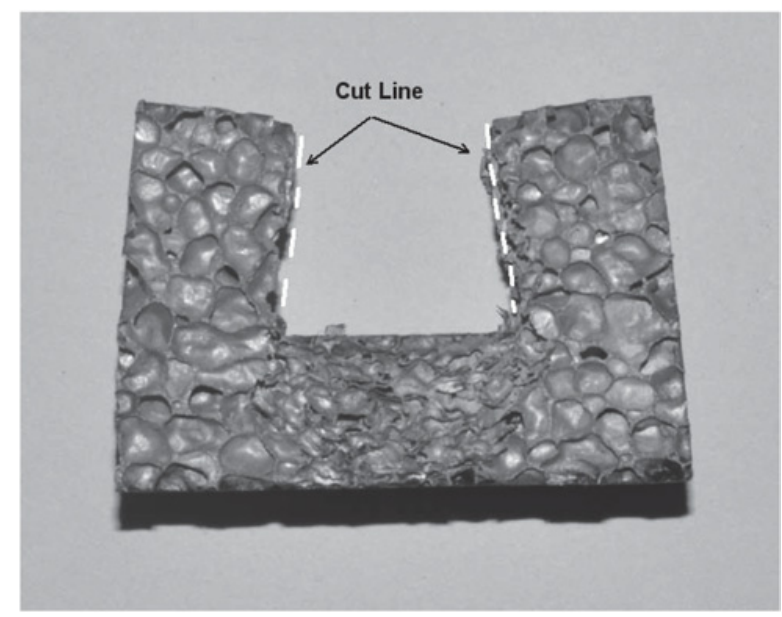

Figure 11. Dynamic indentation specimen after test.

The cells beside the cut lines fractured neatly which means little plasitc energy was dissipated near the cut line. On the other hand, in our previous work [12], no obvious plastic deformation was observed beside the indenter in quasi-static indentaion. As to dynamic indentation, this area should be smaller. So Eq. (15) is used to calculate the tearing energy $\gamma$ in dynamic loading. Then

$$
E_{\text {ind }}=E_{\text {com }}+\frac{2 \gamma}{w}
$$

where $w$ is the width of the indenter. From Fig. 10, it can be observed that the difference between $E_{\text {ind }}$ and $E_{\text {com }}$ does not change much for velocity of $10 \mathrm{~m} / \mathrm{s}-30 \mathrm{~m} / \mathrm{s}$, which means the tearing energy $\gamma$ does not change significantly in this velocity range. Tearing energy $\gamma$ calculated here is $\sim 15 \mathrm{~kJ} / \mathrm{m}^{3}$, which is a little higher than quasi-static value $\sim 12 \mathrm{~kJ} / \mathrm{m}^{3}$ from our previous study [12].

\section{FEM analysis}

To investigate the reason for the increase of the energy absorbed by specimen at velocity of $10 \mathrm{~m} / \mathrm{s}-30 \mathrm{~m} / \mathrm{s}$, an FEM analysis using ABAQUS Explict was conducted. A 3D Voronoi model was used to simulate the compression and indentation process. The geometric information of the voronoi model was obtained from a $\mathrm{C}++$ based program, 


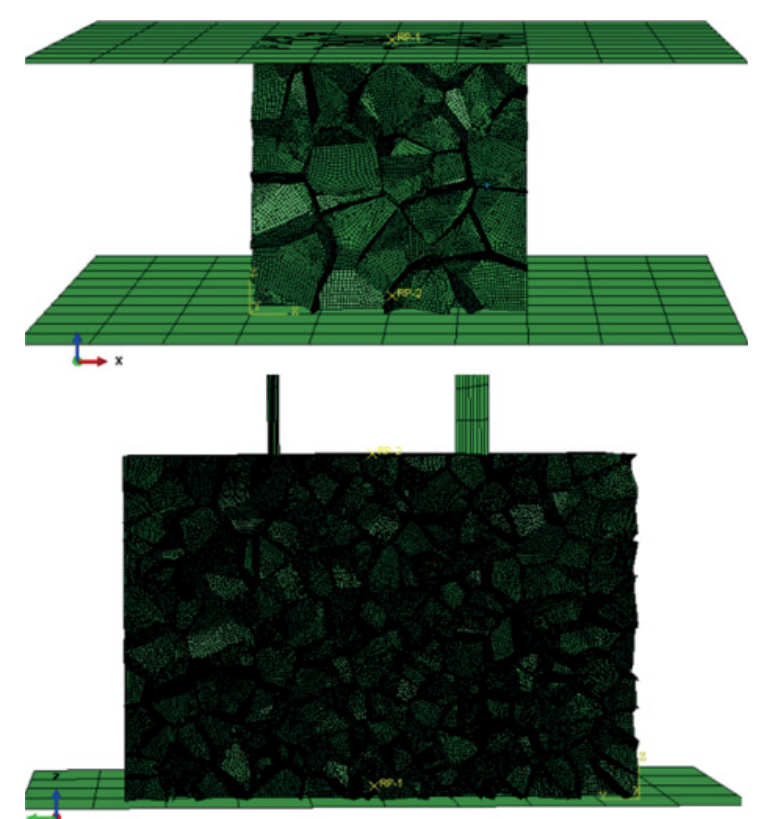

Figure 12. FEM model for compression (above) and indentation (below).

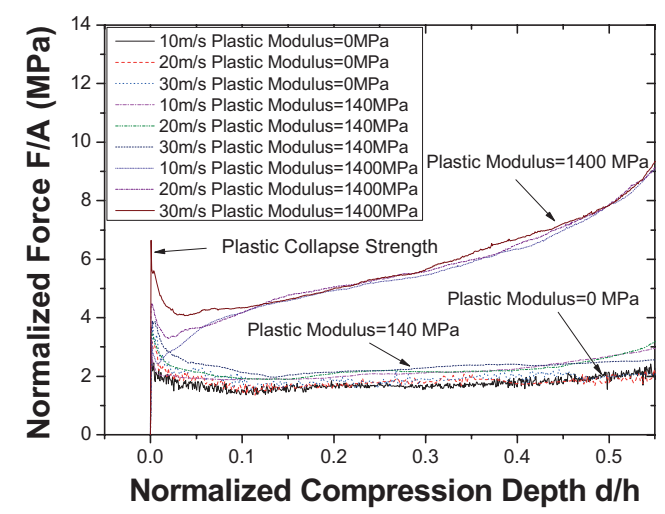

Figure 13. FEM result for compression.

Voro++ [13], and then imported to CAE program to establish an FEM model. The FEM model is shown in Fig. 12, where the indenter and the support was modelled as rigid body. The cell walls were made up of S4R and S3R elements and the thickness of the cell walls was ajusted to fit the mass of the foam specimen. Hard contact was used in the model. No friction and no slide between the foam and the support was considered in our simulation. The compression simulation was firstly conducted. An elasticperfect plastic model and elastic-plastic hardening model were used for cell wall material respectively. The elastic modulus and the yield stress of the material are $69 \mathrm{GPa}$ and $50 \mathrm{MPa}$ respectively. For elastic-plastic hardening model, the two different plastic modulus was set as $140 \mathrm{MPa}$ and $1400 \mathrm{MPa}$. The normalized force as the function of normalized compression depth is shown in Fig. 13. Note the the plastic modulus for elastic-perfect plastic model is 0 .

Neither the plastic collapse strength nor the plateau force of the model with elastic-perfect plastic material increases with increasing velocity, which means that

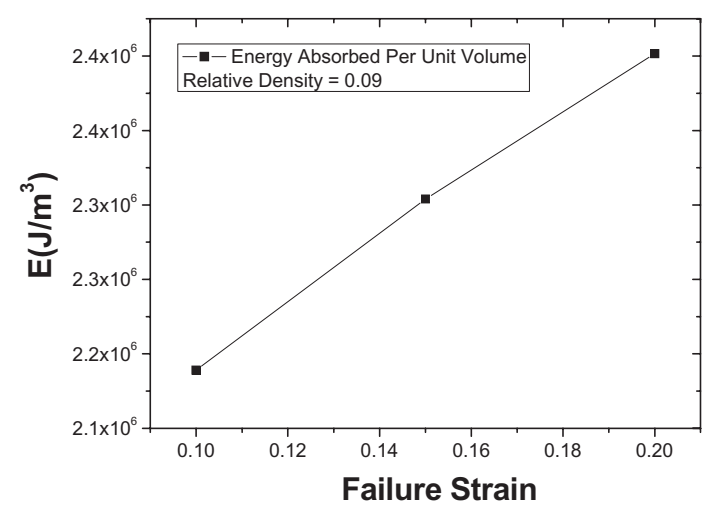

Figure 14. The energy absorbed per unit volume in indentation changing with failure strain.

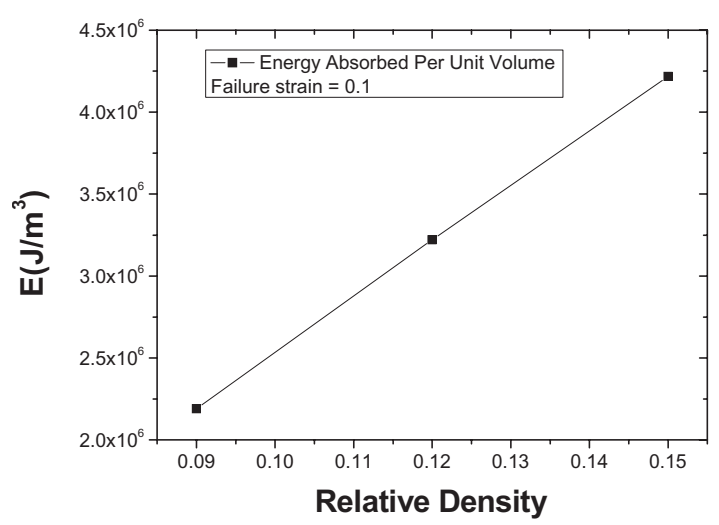

Figure 15. The energy absorbed per unit volume in indentation changing with relative density.

the inertia effects of the cell wall does not dominate in this velocity range. While the plastic hardening model shows great velocity sensitivity about the collaspe strength especially for the model with plastic modulus of $1400 \mathrm{MPa}$. The reason for this is the effect of plastic wave propagated in cell walls. The pasitc wave velocity $C_{p}$ can be expressed as

$$
C_{p}=\sqrt{E_{p} / \rho} .
$$

Where $E_{p}$ is the plastic modulus and $\rho$ is the density of the material. Considering the plastic wave effect, the dynamic plastic collapse stress can be written as

$$
\sigma_{p l}=\sigma_{q s}+\rho C_{p} V_{i} .
$$

Where $\sigma_{q s}$ and $V_{i}$ are quasi-static plastic collapse stress and node velocity of the cell wall. Equation (18) explained the increase of velocity sensitivity with increasing plastic modulus of the material. It should be noted that the plateau strengh of all the models did not show the velocity sensitivity, the reason for this difference from the tests result needs further investigation.

To simulate the indentation process, the elements near the tearing surface were eliminated when the plastic strain reached $\varepsilon_{f}$. The indentation at $10 \mathrm{~m} / \mathrm{s}$ was simulated and different $\varepsilon_{f}$ was used to investigate the relationship between the failure strain and the indentation energy. With failure strain 0.1 , models with relative density of 0.09 , $0.12,0.15$ were also analysed. To simplify the analysis, 
elastic-perfectly plastic material model was used. The results are shown in Figs. 14 and 15. The indentation energy increases linearly with both failure strain of the elements and relative density of the foam. This increase may due to the linear increasing plastic work needed to tear the element with increasing failure strain and cell wall thichness.

\section{Conclusions}

In this paper, the indentation behaviour of aluminum foam at velocity of $10 \mathrm{~m} / \mathrm{s}-30 \mathrm{~m} / \mathrm{s}$ was investigated. SHPB was used to conduct the tests and 3D Voronoi foam model was used to simulate the compression and the indentation process. It was found that the compression and indentation energy absorbed per unit volume change slowly for the loading velocity below $\sim 10 \mathrm{~m} / \mathrm{s}$ and increases significantly at velocity of $10 \mathrm{~m} / \mathrm{s}-30 \mathrm{~m} / \mathrm{s}$. FEM analysis implies different mechanism in these two ranges. The inertia effect of the cell wall dominates at first and then the plastic wave plays an important role. The relationship bewteen the energy aborption ability and the failure strain of the element as well as the relative density of the model was also investiged.Tthere is a linear relationship between the energy absorbed per unit volume in indentaion and both the failure strain of the element and the relative density of the Voronoi model.

The authors would like to thank the financial support by the National Natural Science Foundation of China (Contract no. 11372256) and the 111 Project (Contract no. B07050). Part of this work was conducted when the first author (X.Shi) was attached for two years in Nanyang Technological University, Singapore, under a scholarship from the Chinese Scholarship Council. This paper was also supported by Center for High Performance Computing of Northwestern Polytechnical University, China.

\section{References}

[1] J.A. Reglero, M.A. Rodriguez-Perez, E. Solorzano, J.A. de Saja, Materials \& Design, 32, 2 (2011).

[2] W.H. Hou, F. Zhu, G.X. Lu, D.N. Fang, International Journal of Impact Engineering, 37, 10 (2010).

[3] P.S. Kumar, S. Ramachandra, U. Ramamurty, Materials Science and Engineering a-Structural Materials Properties Microstructure and Processing, 347, 1-2 (2003).

[4] S. Ramachandra, P.S. Kumar, U. Ramamurty, Scripta Mater, 49, 8 (2003).

[5] C. Bacon, Experimental Mechanics, 38, 4 (1998).

[6] V.S. Deshpande, N.A. Fleck, International Journal of Impact Engineering, 24, 3 (2000).

[7] I. Irausquin, J.L. Perez-Castellanos, V. Miranda, F. Teixeira-Dias, Materials \& Design, 47 (2013).

[8] L.J. Gibson, M.F. Ashby, Cellular solids: structure and properties, 2nd ed., Cambridge University Press, Cambridge; New York, 1997.

[9] H. Zhao, I. Elnasri, S. Abdennadher, Int J Mech Sci, 47, 4-5 (2005).

[10] P.J. Tan, S.R. Reid, J.J. Harrigan, Z. Zou, S. Li, Journal of the Mechanics and Physics of Solids, 53, 10 (2005).

[11] A. Paul, U. Ramamurty, Materials Science and Engineering a-Structural Materials Properties Microstructure and Processing, 281, 1-2 (2000).

[12] X.P. Shi, Y.L. Li, G.X. Lu, C.J. Shen, Materials Science and Engineering a-Structural Materials Properties Microstructure and Processing, 614, (2014).

[13] http://math.lbl.gov/vorot+/about.html. 